\title{
Center Research on Insulation Repair Robot Based on STM32 Microcomputer
}

\author{
Zhongxiu Xiao \\ North China Electric Power University, Baoding 071000, China; \\ 1565949101@qq.com
}

Keywords: Inspection Robot, Mechanical Structure, Insulation Repair, Circuit Hardware system.

\begin{abstract}
With the continuous expansion of the size of China's power grid, the traditional inspection line has been difficult to meet the requirements of the future power system, research and application of inspection robot have been become necessary. Instead of manual patrol line, the inspection robot not only can complete insulation repair work of high efficiency and can avoid the unnecessary risk. In this paper, we focus on the study of $10 \mathrm{KV}$ overhead line insulation repair robot. According to the actual situation of construction site, an insulation repair robot is developed and applied in 10KV overhead lines. The ground staff control robot by tablet, control the insulation repair robot and move to the location of repair, then wrap patches on the wire. The whole operation process can be in live line, it realizes completing insulation repair work by robot instead of artificial.
\end{abstract}

\section{Introduction}

The power industry is the most important basic energy industry in the national economic development and the basic industry that relates to national economy and the people's livelihood. With the progress of modern science and technology, automation equipment is widely used in all walks of life. As the traditional energy industry, with the development of new energy sources and the continuous expansion of power grid, power generation and power supply technologies and equipment improvements have caused the daily workload and difficulty of the power industry to increase, and the demand for automation equipment has obviously risen.

In the medium and low voltage $10 \mathrm{kV}$ distribution lines of rural areas overhead lines often encounter such as trees and other variable obstructions. In order to ensure the safety and reliability of the line, measures of wrapping the insulation rubber on the $10 \mathrm{kV}$ distribution line are often adopted to enhance the insulation of the line and ensure the power distribution in an orderly and safe manner.

At present, the insulation repair work by the electric lift truck will be sent to the construction site to complete. This approach is only applicable to the open coastal areas and rapidly developing inland urban areas, which have great limitations and are less and less suited to the needs of modern power grids and the rapid economic and social development. However, with the rapid development of science and technology, as robotics matures, intelligent devices become an important part of the smart grid. Therefore, it is of great significance for us to develop a high voltage insulation repair robot that can be applied to $10 \mathrm{KV}$ lines.

\section{Hardware Introduction}

Currently the majority of domestic and international distribution line on the robot only a simple transmission line alarm function, very few have a line de-icing function. As for the automatic repair of distribution line insulation, so far there is no relevant research papers and patent results and practical application of the product.

And we study the automatic repair of distribution line insulation robot will replace the manual ascension operation wrap the insulation in the fault line to maintain its normal work.

The device consists of walking mechanism, lifting mechanism, wrapping mechanism. Walking agencies mainly constituted by the link between the two driving composition, robot walking is controlled by the transmission between two shots, At the same time, the distance between two wheels 
can be adjusted to adjust the angle of the two connecting rods to control the robot to lift. Wrapping mechanism is the core of the entire robot. Conveying and wrapping insulation rubber is controlled by the connection of rod and pulley.

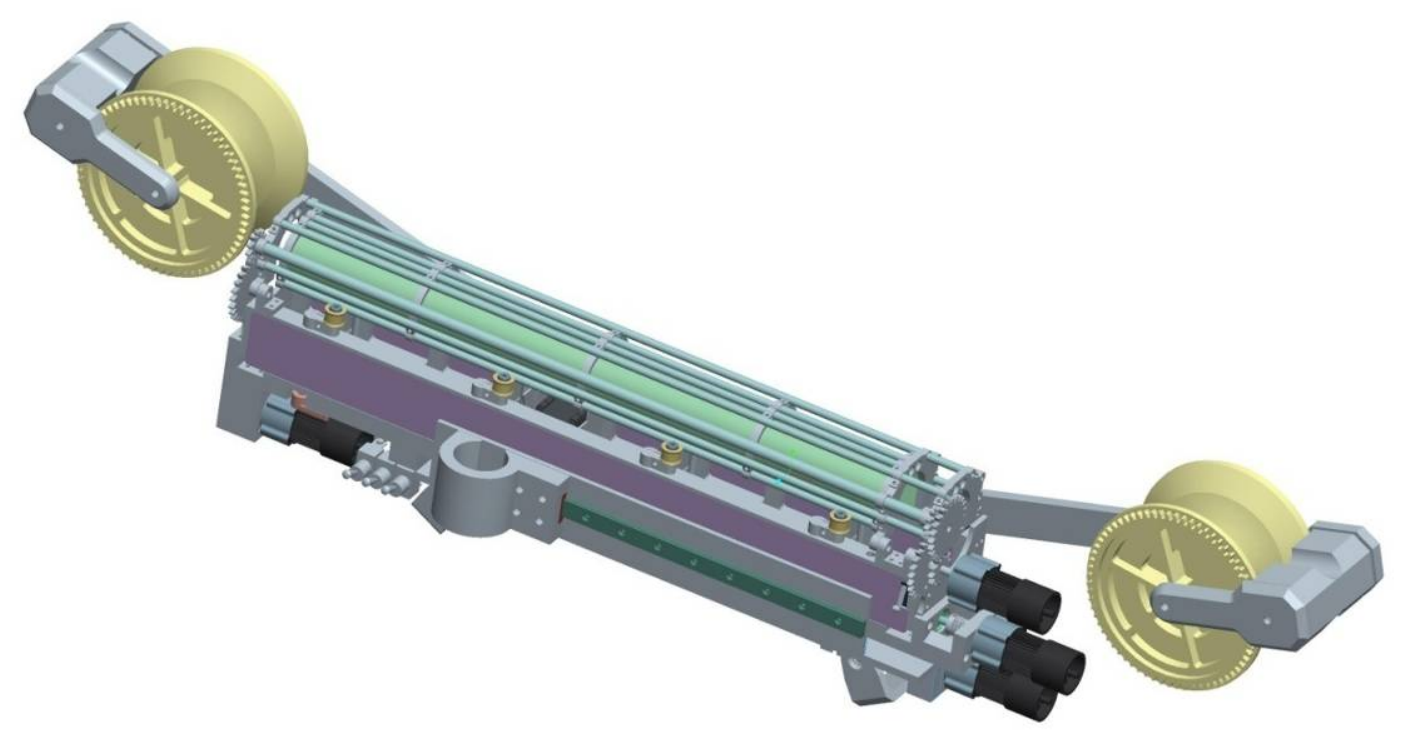

Fig.1 Line insulation repair robot mechanical structure assembly diagram

\subsection{Lifting and Walking Module.}

It is responsible for the whole machine online walking and lifting work. Lifting part is composed of two arms scissors mechanism which is driven by a motor screw, screw driven scissors body bottom so that the angle between the two cantilevers change to control the entire robot to complete the rise and fall movements. The walking part is driven by two motors installed at the top of the scissor mechanism to drive the complete machine to go online. Lifting part of the deformation diagram is as follows:

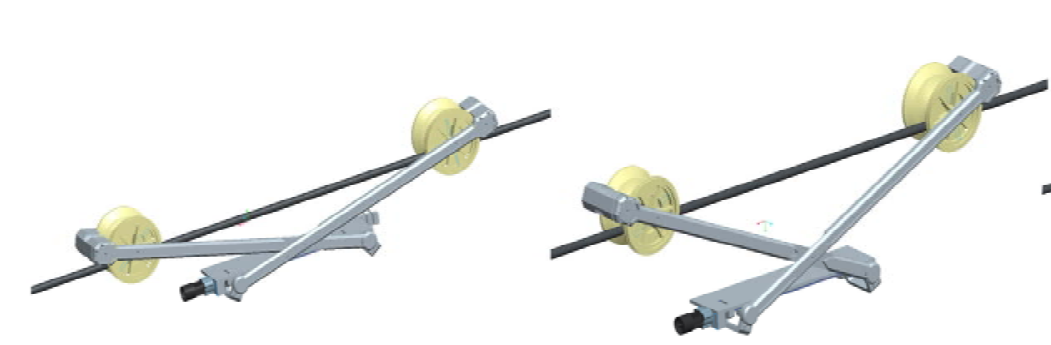

Fig.2 Lifting part of the deformation diagram

\subsection{Wrap Module.}

The wrap module is the body of the robot which is responsible for wrapping cable patch on the cable break. This module is divided into three parts: bracket, manipulator, heating plate. Bracket is used to hold the patch, which itself can be completed open and closed to achieve the main package action. The manipulator is used to clamp the zipper of the cable patch so as to close the open end of the patch. Heaters are used to heat the patches which are made of electric original. The piece to be heated has been wrapped around the cable after the zipper closed, then start the electric heating plate so that repair patches will shrink and tightly wrapped in the cable damage. 

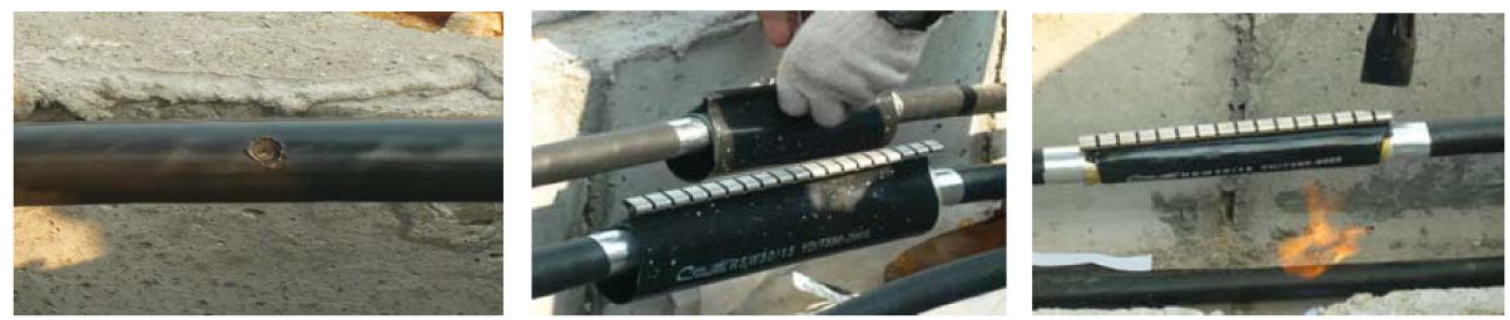

Fig.3 Method of using cable patch.

\subsection{Control Chip.}

Distribution line insulation repair device can be controlled by the following ways: 1. PC software; 2. mobile client. Users can control the insulation repair device anytime, anywhere. It is safe and reliable, more convenient and faster. It also avoids unexpected events that occur during automatic operation.

The core of our product control is the STM32 microcontroller, which is responsible for receiving PC (computer-side) information and send control information to the motor, while receiving the signal sent by the Bluetooth module. STM32 microcontroller is of stable performance and good control, the entire microcontroller is highly integrated. Compared to the widespread use of 51 Microcontroller it has greatly improved.

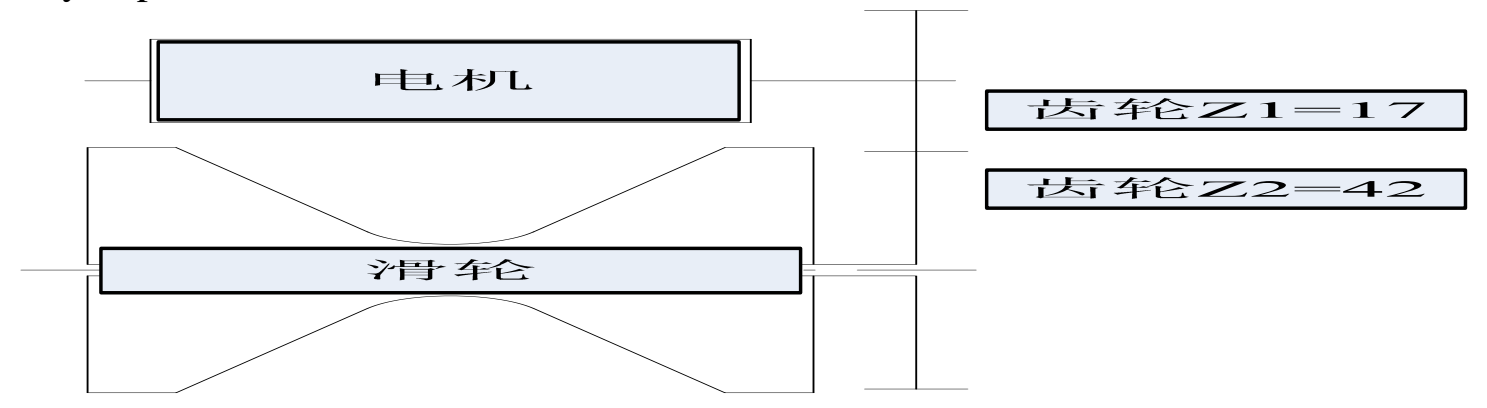

Fig.4 Tablet computers and line insulation repair robots.

\section{Working Steps}

When the robot performs the package task, the workflow is designed as follows:

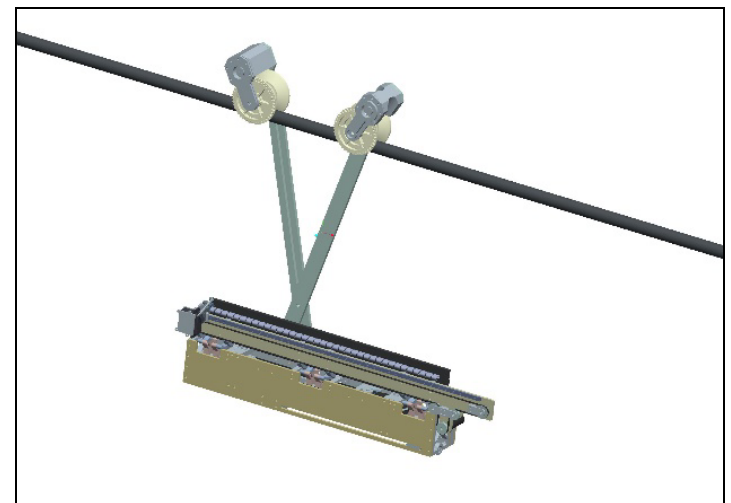

Fig.5 select the end near

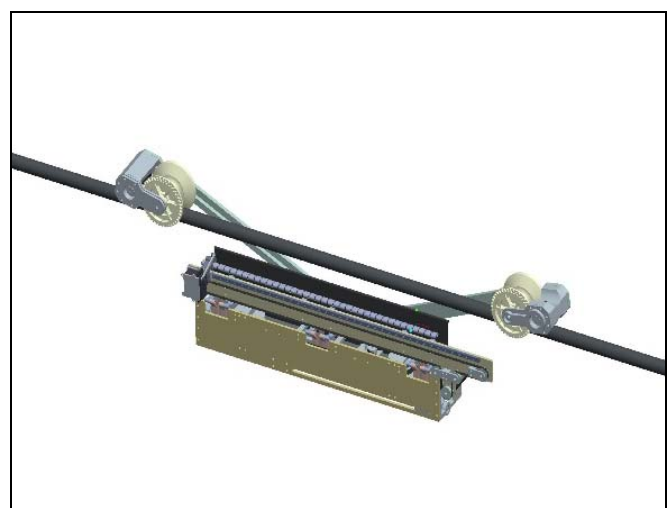

Fig.6 the robot to send wire

Arrived at the construction site, select the end near the pole to facilitate the robot to send wire. Be prepared on the ground, such as power inspection, communication check, etc. Install the cable patch in the robot bracket and adjust the position of the robot. At this time, the robot is in suspension, and the two arms are open to the minimum angle, which is convenient for suspension.

Use two insulated poles to send the insulated repair robot to the cable. Two insulation poles, one for orientation, one for thrust. Hang the driving pulleys of two cantilever arms of the robot on the overhead wires. Through the tablet control two arms open a larger angle. The robot is in a walking state, then take off the insulation rod. 
Through tablet computer remote control the insulation repair robot. After the robot walks to the cable breaking point, execute package to wrap cable patch on cable break, then draw the zip, electric heating seal. After the package is completed, the robot returns to walking status. The remote-controlled robot pulls away from the cable and returns to the end of the pole.

Use two insulated rods to remove the insulated repair robot from the cable. Two insulation poles, one for orientation, one for thrust. Through the tablet control two arms open the minimum angle. At this point the robot is in a suspended state, remove the robot from the cable, withdraw the insulation rod and complete the operation.

\section{Summary}

The robot we designed has the following innovations. Mechanical structure innovation: to get rid of the complicated structure of traditional robot, the robot can walk and hang in many states with simple cutting fork structure. Control mode innovation: it can be controlled remotely through mobile APP and power supply company control center; it can automatically control the whole, manual control of local. It is the first domestic insulation repair automation process.

The interconnection of equipment is reflected in the following aspects. Data collection and analysis: infrared thermography and camera and other sensors are added to the basic robot to collect and analyze data such as line temperature. Real-time sharing of information: the robot has a strong communication capability, which can be connected with customer control center, computer, mobile phone, etc. to realize multi-terminal operation and real-time sharing of data. Feedback of work: we developed the equipment testing program, and periodically checked the working condition of the equipment and gave feedback to us so as to provide maintenance and service. However, the robot designed by this project has limited climbing ability. And when the wire is climbing a larger slope, the robot still needs improvement.

\section{References}

[1]. J.Sawada, K.Kusumoto, T.Munakata. A mobile robot for inspection of power transmission lines.IEEE Transactions on Power Delivery. 1991, 6(1), p.309-315.

[2]. Wu Peng:Development of Insulation Repair Robot for 10KV Overhead Line.p.27-56.

[3]. L.Tang, L.J.Fang, H.G.Wang. Development of an inspection robot control system for 500kV extra high voltage power transmission lines. The Society of Instrument and Control Engineers Annual Conference.Tokyo, Japan: SICE, 2004, p.329-334.

[4]. Sato Kensetsu Kogyo Co., Ltd. Automatic Overhead Power Transmission Line Damage Detector. Website(http://www.sato-k.co.jp)

[5]. Rosenfeld.Y, Logcher. R. D. New Concepts for Deployable Collapsible Structures [J]. International Journal of Space Structures, 1998.3(1), p.20-32. 